\title{
Selen in der Intensivmedizin
}

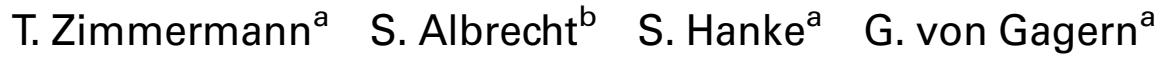 \\ ${ }^{a}$ Klinik für Viszeral-, Thorax- und Gefäßchirurgie, ${ }^{b}$ Klinik und Poliklinik für Gynäkologie und Geburtshilfe, Universitätsklinikum Carl \\ Gustav Carus, Technische Universität Dresden
}

\section{Schlüsselwörter}

Selen · Selenoenzyme · Transkriptionsfaktoren · Sepsis · SIRS

\section{Zusammenfassung}

Mit der Entdeckung des Selens als essentielles Spurenelement und der Glutathionperoxidase als Selenoenzym sowie der Tatsache, daß Selenmangel relativ weit verbreitet ist, wurde erstmals ein Zusammenhang zu einigen schweren Erkrankungen hergestellt (Keshan-Krankheit, Kaschin-Beck-Syndrom). Interessant ist dabei, daß sich trotz dieser und anderer bekannter Selenmangelerkrankungen eine Therapie in der Humanmedizin nur langsam zu etablieren beginnt, während die Selensupplementation in der Veterinärmedizin bereits Standard ist. Die Autoren beschäftigen sich seit 1990 mit der Rolle des Spurenelements Selen bei septischen Krankheitsbildern in der Intensivmedizin, beim Reperfusionsphänomen nach gefäßchirurgischen Eingriffen und in der Onkologie. Sie konnten zeigen, daß die adjuvante Therapie der akuten Pankreatitis und der Sepsis mit Natriumselenit einen positiven Effekt auf das Outcome der Patienten zu haben scheint (eine multizentrische, doppelblinde, randomisierte Sepsisstudie zur Validierung dieser Ergebnisse ist in Vorbereitung). Neue Erkenntnisse zur Beeinflussung von Transkriptionsfaktoren durch Selen bei systemischem Inflammationssyndrom und Sepsis erlauben eine wissenschaftlich fundierte Interpretation der klinischen Ergebnisse. Weitere molekularbiologische Untersuchungen werden das Spurenelement Selen zu einem der interessantesten Forschungsprojekte der nächsten 10 Jahre in Intensivmedizin und Onkologie machen.

\section{Einleitung}

1957 entdeckte Schwarz [1] die Essentialität des Selens. Auch die Glutathionperoxidase (GSH-Px) wurde bereits 1957 beschrieben, aber erst 1973 von Rotruck et al. [2] als Selenoenzym erkannt.

\section{KARGER}

Fax +497614520714

E-mail Information@Karger.de wwwkarger.com/journals/cga

(c) 2000 S. Karger GmbH, Freiburg

Accessible online at:

www.karger.com

\section{Key Words}

Selenium · Selenoenzymes · Transcription factors $\cdot$ Sepsis $\cdot$ SIRS

\section{Summary}

Since selenium was discovered as an essential trace element being widely distributed, and since glutathione peroxidase is known as a selenoenzyme, associations with several severe diseases were established (Keshan disease, Kaschin-Beck syndrome). Despite these known selenium deficiency diseases a related human therapy is still not established so far. In veterinary medicine, however, substitution of selenium is already a standard therapy. Our laboratory investigates the role of selenium since 1990. This includes investigations about the effects of selenium in acute inflammatory diseases in intensive care, in the reperfusion phenomenon following vascular surgery, and in oncology. In acute pancreatitis and sepsis, adjuvant therapy using sodium selenite seems to have positive effects on the overall outcome of patients (a multicenter, double-blind, randomized trial on sepsis is being prepared). New findings concerning the influence of selenium on transcription factors in inflammatory processes will permit a scientifically sound interpretation of clinical results. With further investigations in molecular biology the trace element selenium will become, in the next decade, one of the most interesting topics in intensive care and oncology.

Beim Menschen sind bislang 4 GSH-Px nachgewiesen. Weitere Selenoenzyme sind das Selenoprotein P, die Deiodasen vom Typ I, II und III und auch die Thioredoxinreduktase, ein Flavoenzym, welches einen Selenocysteinrest enthält. Die GSH-Px schützen den Körper gegen schädigende Oxidationsvorgänge, indem sie sowohl 
Tab. 1. Wirkungsprofil der GSH-Px

\begin{tabular}{|c|c|}
\hline Peroxidasen & Wirkung \\
\hline c Gp & $\begin{array}{l}\text { ubiquitär } \\
\text { Lokalisation in Zytosol und Mitochondrien } \\
\text { Schutzenzym gegen oxidativen Streß unter pathologi- } \\
\text { schen Bedingungen }\end{array}$ \\
\hline gi Gpx & $\begin{array}{l}\text { Schutzwall gegen Aufnahme peroxidierter Nahrungs- } \\
\text { bestandteile }\end{array}$ \\
\hline p Gpx & $\begin{array}{l}\text { Abpufferung der physiologischen Schwankungen des } \\
\text { extrazellulären Peroxidtonus }\end{array}$ \\
\hline PH Gpx & $\begin{array}{l}\text { Zellreifung, Zytokinbildung, Phagozytose } \\
\text { (Reduktion der Produkte der 15-Lipoxigenase) }\end{array}$ \\
\hline
\end{tabular}

Wasserstoffperoxid als auch Lipid-, Steroid- und DNA-Peroxidase reduzieren [3, 4]. Die Wirkungsweise der einzelnen GSH-Px ist in Tabelle 1 dargestellt.

GSH-Px katalysieren die Übertragung von Wasserstoff und Glutathion auf Peroxide. Bei der Reaktion dieser Peroxide entstehen Wasser und Alkohole. Der Selenrest wird oxidiert und von Glutathion wieder reduziert. Die GSH-Reduktase regeneriert das oxidierte Glutathion. Dieser Prozeß geschieht auf Kosten von $\mathrm{NADPH}+\mathrm{H}^{+}$.

Die Bedeutung der GSH-Px bei Entzündungsvorgängen ist hinlänglich beschrieben worden [5]. Eine verminderte GSH-Px-Aktivität führt bei einem massiven Anfall von Sauerstoffradikalen zur verstärkten Bildung von Metaboliten der Arachidonsäurekaskade (Prostaglandine, Leukotriene, Lipidperoxide). In vivo und in vitro konnte gezeigt werden, daß die Supplementation mit Natriumselenit sowohl zu einem Anstieg der Selenkonzentration als auch einer Steigerung der GSH-Px-Aktivität führen kann.

Kritiker berichten jedoch, daß zur Biosynthese der Selenoproteine Zeit benötigt wird und sich daher nicht alle Selenwirkungen über die Selenoenzyme erklären lassen, da die Biosynthese der Selenoproteine einer hierarchischen Ordnung unter selenlimitierten Bedingungen unterliegt [6].

Das Selenoenzym Thioredoxinreduktase ist gemeinsam mit dem Thioredoxin wichtig zur Aufrechterhaltung des antioxidativen Potentials einer Zelle. Weitere selenabhängige Enzyme sind die Iodthyronin-5'-deiodasen (ID 1, 2, 3). Diese Iodthyronin-5'-deiodasen scheinen eine Schlüsselrolle im Schilddrüsenstoffwechsel zu spielen. Das Selenoprotein P nimmt möglicherweise einen wichtigen Platz im plasmatischen antioxidativen Schutzmechanismus ein.

Erst 1991 wurde durch die Deutsche Gesellschaft für Ernährung (DGE) eine Aufnahme von 20-100 $\mu$ g Selen täglich empfohlen. 1996 konnten Clark et al. [7] nach Supplementation mit $200 \mu \mathrm{g} \mathrm{Se}$ lenhefe einer Verringerung der Tumorinzidenz und -mortalität von Kolon-, Lungen- und Prostatakarzinomen erreichen. 180 Jahre nach Entdeckung des Selens wurde in zwei unabhängig voneinander durchgeführten Pilotstudien eine deutliche Mortalitätssenkung bei Patienten mit systemischem Inflammationssyndrom (SIRS) oder Sepsis erreicht $[8,9]$.

Obwohl die Selensupplementation in der Veterinärmedizin zur Therapie von verschiedenen Erkrankungen (Lebernekrose, Weißmuskelkrankheit, exsudative Diathese) bereits erfolgreich einge- setzt wird, fürchten sich noch viele Humanmediziner vor einer therapeutischen Gabe von Selen aus Angst vor dem scheinbaren «engen therapeutischen Fenster». Mit immer neuen Erkenntnissen über das Spurenelement Selen und vor allem neuesten molekularbiologischen Daten steigt die Gewißheit, die «sichere Dosis» zu applizieren.

\section{Anwendung von Selen in der Intensivmedizin}

In den vergangenen Jahren wurden auf der Suche nach neuen Therapiestrategien bei SIRS/Sepsis-Patienten, die nach wie vor eine Letalität $>40 \%$ aufweisen, zwei Wege eingeschlagen: zum einen die Eliminierung des Endotoxins und zum anderen die Eliminierung bzw. Hemmung der Mediatoren. Anfang der 90er Jahre machte sich eine große Enttäuschung breit, die nach wie vor anhält. Während tierexperimentelle Untersuchungen zur Mediatorenblockade gute Ergebnisse zeigen, versagen diese Therapieansätze in Phase-III-Studien zumeist. 30 Jahre Mediatorenforschung führten zu einem Mediatorenwirrwarr ohne therapeutische Konsequenzen. Das war Anlaß für unsere Arbeitsgruppe, nach neuen Konzepten und Möglichkeiten zu suchen. Anfang 1990 berichteten viele Autoren über einen stark erniedrigten Selenspiegel bei schwerkranken Patienten auf Intensivstationen. Die Ursache war unklar. Eine Selenmangelversorgung wurde diskutiert, aber auch, daß Selen zu Beginn schwerer systemischer Erkrankungen rasch im Körper umverteilt wird und es zu einer höheren Ausscheidung über die Niere kommt. Die parenterale Ernährung verstärkte den bereits bestehenden Selenmangel. Patienten mit SIRS/Sepsis und/ oder Pankreatitis zeigten eine deutlich vermehrte Radikalbildung. Demgegenüber stand ein durch Selenmangel geschwächtes Redoxsystem, in deren Folge es zu Organschäden kommen kann. Dies führte zu der Überlegung, Selen in Form des Natriumselenits als Therapeutikum einzusetzen.

\section{Selen und akute Pankreatitis}

Seit 1990 appliziert Kuklinski [10] in Rostock Natriumselenit zur Therapie der akuten Pankreatitis. Er konnte in der 1. Phase eine Letalitätssenkung auf $0 \%$ erreichen, so daß er nach 18 Patienten die als randomisierte Studie geplante Untersuchung abbrach und alle Patienten seit dieser Zeit mit Natriumselenit behandelt. In Auswertung eines größeren Krankenguts konnte er dieses Ergebnis nicht mehr erreichen. Die Letalität blieb jedoch nach wie vor deutlich unter der einer historischen Verlaufskontrolle.

In Dresden begannen wir im Mai 1991 mit der Natriumselenitgabe bei Patienten mit akuter Pankreatitis. Alle Patienten erhalten initial $1000 \mu \mathrm{g}$ Natriumselenit als Bolus zur Aufsättigung bei nachgewiesenem extremem Selenmangel und danach über $24 \mathrm{~h} 1000 \mu \mathrm{g}$ als kontinuierliche Infusion. Diese Therapie wurde bis zur Normalisierung der Entzündungsparameter fortgesetzt (Normalisierung von C-reaktivem Protein, Leukozyten, Elastase). Die anfängliche Randomisierung im Rahmen einer klinischen Studie wurde eben- 
falls nach 18 Patienten abgebrochen. In Auswertung des Krankenguts der letzten 10 Jahre (216 Patienten mit akuter Pankreatitis) konnten wir zu einer historischen Vergleichsgruppe (1986-1991 versus 1991-1996) den positiven Effekt der Natriumselenittherapie nachweisen. Wir erzielten eine deutliche Senkung der Frühletalität (7-, 14-, 28-Tage-Letalität). In der Zusammenschau aller Patienten konnten wir feststellen, daß Patienten, die initial sofort eine Natriumselenittherapie erhielten, eine Letalität von $4,2 \%$ aufwiesen. Dagegen verstarben in der Patientengruppe, die erst sekundär (von außerhalb zugewiesen, teilweise mit Organversagen) eine Natriumselenittherapie erhielten, $16 \%$ der Patienten, so daß die Gesamtletalität in der Selenitgruppe (primäre und sekundäre Applikation) $10,1 \%$ betrug. Nicht berücksichtigt wurde bei dieser Auswertung die deutliche Verbesserung der Intensivtherapie («aggressive intensivtherapeutische Maßnahmen») und der Wandel des Therapiekonzepts hin zur konservativen Therapie, die auch eine Letalitätsverbesserung erbrachte. Eine Multizenterstudie zur Therapie der akuten Pankreatitis bezüglich der Wirksamkeit der Selenittherapie soll und muß erfolgen.

\section{Selen und Sepsis}

Eine «mediatorengerechte» Therapie der Sepsis existiert bislang nicht. Die Schwierigkeit, eine «Multimediatorenkrankheit» wie die Sepsis mediatorengerecht $\mathrm{zu}$ behandeln, besteht darin, daß die Labordiagnostik letztendlich nur bereits abgelaufene pathobiochemische Prozesse beschreibt. Eine Möglichkeit, in der Kaskade wirksam einzugreifen, scheint hierbei die Inhibierung der Endprodukte, d. h. der reaktiven Sauerstoffradikale, zu sein. Sauerstoffradikale werden sowohl als Reaktionsprodukt der Aktivierung der Kaskadensysteme und des Arachidonsäuremetabolismus als auch von Granulozyten, Monozyten und im hypoxischen Gewebe (Reperfusionsschaden) gebildet. Sie stimulieren wiederum den Arachidonsäuremetabolismus mit Bildung proinflammatorischer Produkte, wirken chemotaktisch, schädigen direkt das Endothel, zerstören Antiproteasen und initiieren die Lipidperoxidation. Sauerstoffradikale greifen auch auf molekularer Ebene in zentrale Schaltstellen der inflammatorischen Reaktion ein, wie z. B. in die Aktivierung des redoxsensitiven Transkriptionsfaktors NF- $\kappa \mathrm{B}$ in mononukleären Blutzellen. Patienten mit SIRS/Sepsis weisen eine Mortalitätsrate von etwa $40 \%$ auf. In zwei prospektiv randomisierten monozentrischen Studien wurde gezeigt, daß zusätzlich zu dieser üblichen intensivmedizinischen Therapie bei schwerkranken Patienten (APACHE-II-Score $\geq 15$ ) eine Substitution von Selen tendenziell zu einer Reduktion der Mortalität führt.

\section{Selensubstitution bei Sepsispatienten}

\section{Dresdner Studie}

In der Klinik und Poliklinik für Viszeral-, Thorax- und Gefäßchirurgie Dresden wurden 40 Patienten, die den Kriterien des SIRS/ Sepsis entsprachen, in die Studie aufgenommen. 20 Patienten erhielten anhand einer Randomisierungsliste eine Substitution mit Natriumselenit. Die Patienten der Verum-Gruppe erhielten am
1. Tag $1000 \mu \mathrm{g}$ Natriumselenit als Bolus sowie vom 1. bis zum 14. Tag $1000 \mu \mathrm{g}$ Selen/24 h als kontinuierliche Infusion. Den Patienten der Kontrollgruppe wurde kein zusätzliches Selen verabreicht. Die Mortalität konnte dabei von $40 \%$ in der Kontrollgruppe auf $15 \%$ in der Natriumselenitgruppe reduziert werden. Als klinische Verlaufsparameter wurden der APACHE-II-Score und der MOF-Score nach Goris benutzt. Alle Patienten zeigten initial hinsichtlich Schwere der Sepsis und des Organversagens (APACHEII-Score $=15$ Punkte, MOF-Score $=7$ Punkte) keinerlei Unterschiede. Bereits am 2. Tag fielen in der Patientengruppe mit adjuvanter Natriumselenittherapie der APACHE-II-Score und der MOF-Score signifikant ab. Auch die inflammatorische Reaktion [Abfall der Interleukin-6- und der Tumor-Nekrose-Faktor(TNF)alpha-Konzentration im Blut] konnte bereits ab dem 4. Tag nachgewiesen werden. Unbeeinflußt blieb dagegen die PMN-7-Elastase- und Neopterinfreisetzung. Die Selenkonzentrationen in Blut und Plasma stiegen am 2., die GSH-Px-Aktivität bereits am 3. Tag an.

\section{Münchner Studie}

Auf der Internistischen Intensivstation des Klinikums der LudwigMaximilian-Universität München wurden 42 Patienten mit den klinischen und laborchemischen Zeichen einer SIRS/Sepsis und einem APACHE-II-Score $\geq 15$ am Aufnahmetag in die Studie aufgenommen. 21 Patienten erhielten Natriumselenit in geringerer Dosierung als in der Dresdner Studie. Der Kontrollgruppe wurden täglich $35 \mu \mathrm{g}$ Selen im Rahmen einer Spurenelementlösung infundiert. Die Mortalität sank von 55 auf 33\%. Interessant war dabei, daß vor allem schwerkranke Patienten, d.h. mit einem APACHEII-Score $>20$ mit jeweils 10 Patienten in beiden Gruppen, einen noch größeren Gewinn durch die adjuvante Selenittherapie hatten. Hier sank die Mortalität von 70 auf $33 \%$. In Auswertung beider Pilotstudien wurde eine Phase-III-Studie initiiert, die im Jahr 1999 begonnen hat.

\section{Selen und Regulierung der inflammatorischen Antwort auf molekularer Ebene}

Zentraler Regulator der inflammatorischen Antwort des Organismus scheint der redoxsensitive Transkriptionsfaktor NF- $\kappa \mathrm{B} z u$ sein. Dessen überschießende Aktivierung durch TNF-alpha, Phospholipase A2 und reaktive Sauerstoffspezies (ROS) führt zur unkontrollierten inflammatorischen Antwort mit Aktivierung von «immediate early genes» und Ausschüttung von proinflammatorischen Zytokinen. Es konnte gezeigt werden, daß Selen in vitro auf $\mathrm{NF}-\kappa \mathrm{B}$ inhibierend wirkt. In-vivo-Effekte von Selen auf redoxsensitive Transkriptionsfaktoren könnten somit auch einen Teil der positiven Effekte bei Sepsis erklären. Ein weiterer redoxsensitiver Transkriptionsfaktor, der vor allen Dingen in Korrelation mit der inflammatorischen Reaktion in der Leber (Leber als Syntheseort pro- und antiinflammatorischer Mediatoren) steht, ist der Faktor AP-1. Auf diesen Faktor wirkt Selen aktivierend, d. h. proinflammatorisch. Auch AP-1 wird durch Interleukin-6, TNF-alpha und 


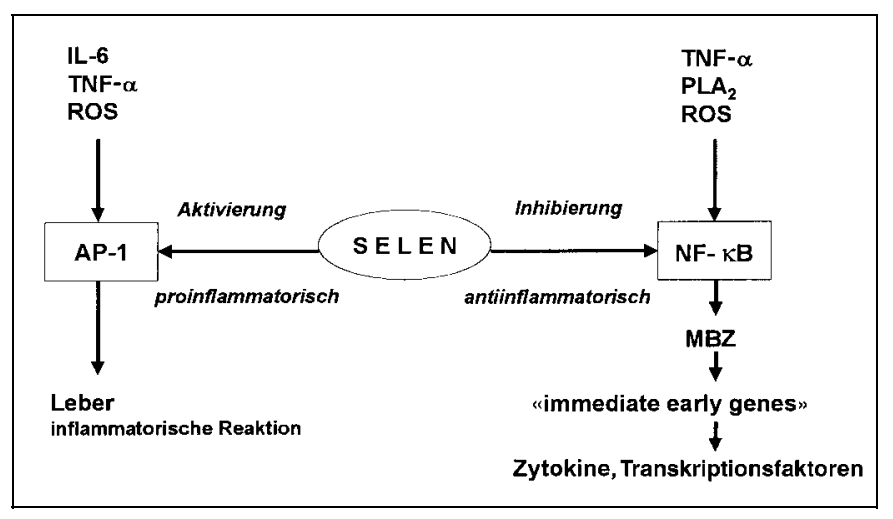

Abb. 1. Möglicher Einfluß von Selen auf die Aktivierung von Transkriptionsfaktoren.

reaktive Sauerstoffspezies aktiviert. Das Redoxgleichgewicht in der Zelle ist dabei von entscheidender Bedeutung (Abb. 1).

In der multizentrischen Phase-III-Studie (SIC-Studie) wird deshalb die Regulierung der inflammatorischen Reaktion durch Selen am Beispiel der zwei Faktoren AP-1 und NF- $\kappa$ B untersucht. Dabei geht es um die Frage, inwieweit eine Störung des Gleichgewichts zwischen beiden Faktoren zu einer nicht mehr beherrschbaren inflammatorischen Reaktion führen kann. Ob dabei AP-1 die kontrollierte Freisetzung von Mediatoren beeinflußt und NF- $\kappa$ B verantwortlich ist für die unkontrollierte Ausschüttung von Mediatoren, soll Ziel dieser Studie sein.

In der Dresdner Sepsisstudie konnte bereits gezeigt werden, daß es bei Patienten, die Natriumselenit als adjuvante Therapie erhielten, zu einem Verschwinden von NF- $\kappa$ B im Zellkern von mononukleären Zellen kommt und daß bei Patienten ohne Selensubstitution aktiviertes NF- $\kappa$ B im Zellkern persistierte mit konsekutiver, nicht beeinflußbarer inflammatorischer Reaktion. Im Rahmen einer Polytraumastudie konnten bereits erste Anhalte dafür gefunden werden, daß der Selenspiegel mit der Aktivierung von NF- $\kappa \mathrm{B}$ und AP-1 korreliert und daß die beiden Transkriptionsfaktoren gegensinnig verlaufen. Überwiegt die Aktivität von AP-1 in mononukleären Zellen, ist die inflammatorische Antwort des Organismus geringer ausgeprägt, währenddessen hohe NF- $\kappa$ B-Bindungsaktivitäten in mononukleären Zellen für eine massive inflammatorische Reaktion sprechen, die durch AP-1 nicht gegenreguliert werden kann. Niedrige Selenspiegel führten zu einer signifikant stärkeren $\mathrm{NF}-\kappa \mathrm{B}-$ Aktivierung als umgekehrt.

\section{Selen und Polytrauma}

1996 wurde von Berger et al. [11] das sogenannte Low-T3-Syndrom bei Polytraumapatienten beschrieben. Inwieweit es sich dabei um ein Phänomen oder einen wichtigen pathogenetischen Faktor handelt, ist bislang noch nicht geklärt. Es ist bekannt, daß Selen in den Schilddrüsenstoffwechsel über die Deiodinasen eingreift. Auch gibt es zahlreiche Literaturhinweise dafür, daß das Low-T3Syndrom mit dem Ausbleiben der hyperdynamischen Phase in der Sepsis und der schlechteren Prognose korreliert. Weitere Studien sind notwendig, um dieses Phänomen abzuklären.

\section{Schlußfolgerungen}

Selen stellt in der Intensivmedizin nicht nur ein Spurenelement dar, welches in der parenteralen Ernährung substituiert werden muß, sondern die Therapiestrategien gehen über moderne Ernährungsstrategien hinaus. Wie die neuesten molekularbiologischen Untersuchungen zeigten, ist dabei nur ein Teil der positiven Effekte einer Selenitsubstitution auf die bekannten Selenoenzyme zurückzuführen. Die Beeinflussung der inflammatorischen Antwort auf Transkriptionsebene über die Transkriptionsfaktoren AP-1 und NF- $\kappa$ B scheint dabei ein weiterer wichtiger Faktor zu sein. Die prospektive randomisierte multizentrische Phase-III-Studie bei Patienten mit SIRS/Sepsis zum Vergleich einer Substitutionstherapie mit $1000 \mu \mathrm{g}$ Selen i.v. versus Placebo, die Ende 1999 begann, könnte einen wichtigen Fortschritt in der Sepsisbehandlung bringen. Man muß jedoch bei der Anwendung von Selen immer noch zwischen Ernährungsprävention und Pharmakotherapie unterscheiden. Für die Ernährung gilt nach wie vor die Empfehlung der DGE mit 20-100 $\mu \mathrm{g} / \mathrm{Tag}$. In der Prävention, d. h. in der Karzinomprophylaxe, sind Dosen zwischen 200 und $400 \mu \mathrm{g} / \mathrm{Tag}$ zu diskutieren. In der Pharmakotherapie ist diese Dosis höher. Sie beträgt $1000 \mu \mathrm{g} / \mathrm{Tag}$, sollte jedoch nur über kürzere Zeiträume gegeben werden. Dosisfindungstudien sind dazu notwendig. Inwieweit ein ausgeglichener Selenstatus eine Rolle in der Prophylaxe bestimmter Krankheiten spielt, ist noch im experimentellen Stadium. Wahrscheinlich werden in Zukunft die Empfehlungen für die tägliche Selenzufuhr als auch für eventuelle therapeutische Strategien nach oben korrigiert werden müssen.

\section{Literatur}

1 Schwarz K, Foltz CM: Selenium as on integral part of factor 3 against dietary necrotic liver degeneration. J Am Chem Soc 1957;79:3292-3293.

2 Rotruck JT, Pope AL, Ganther ME, Swanson AB, Hafeman DG, Hockstra WG: Selenium: Biochemical role as a component of glutathione peroxidase. Science 1973;179:588-590.

3 Siems WG, Krämer K, Gmne T: Zur Chemie und Biologie des Glutathionssystems: ein Überblick. Pharm Z 1996;141:4239-4248.

4 Fox JM: Selenium: Nutritional implication and prospects for therapeutic medicine. Med Find Exp Clin Pharmacol 1992;14:275-287.
5 Reddy CL, Massaro EJ: Biochemistry of selenium: A brief overview. Fund App. Toxicol 1983;3:431-436.

6 Flohe' L, Wingender R, Bringelius-Flohe' R: The regulation of glutathione peroxidasis; in Forman $\mathrm{U}$, Cadomas L (eds): Oxidative Stress and Signal Transduction. London, Capman and Hall (in press).

7 Clark LC, Combs GF Jr, Turnbull BW: The nutrional prevention of cancer with selenium 1983-1993: A randomized clinical trial. JAMA 1996;276:1957-1963.

8 Zimmermann T, et al: Selensubstitution bei Sepsispatienten. Med Klin 1997;92(suppl 3):3-4.
9 Gärtner R, et al: Selensubstitution bei Sepsispatienten. Med Klin 1997;92(suppl 3):12-14.

10 Kukliniski B, Buchner M, Müller T, Schweder R: Antioxidative Therapie der Pankreatitis - eine 18monatige Zwischenbilanz. Z Ges Inn Med 1992;47:229-235.

11 Berger MM, Lemarchand-Beraud T, Cavadini C, et al: Relations between the selenium status and the low T3 syndrome after major trauma. Intensive Care Med 1996;22:575-581. 